\title{
Eskolemization in intuitionistic logic
}

\author{
Matthias Baaz* \\ Inst. for Discr. Math. and Geom. \\ Technical University Vienna \\ Wiedner Hauptstrasse 8-10 \\ 1040 Vienna, Austria \\ baaz@logic.at
}

\author{
Rosalie Iemhoff \\ Dept. of Philosophy \\ University Utrecht \\ Heidelberglaan 8 \\ 3584 CS Utrecht, The Netherlands \\ Rosalie.Iemhoff@phil.uu.nl
}

April 3, 2009

\begin{abstract}
In [2] an alternative skolemization method called eskolemization was introduced that is sound and complete for existence logic with respect to existential quantifiers. Existence logic is a conservative extension of intuitionistic logic by an existence predicate. Therefore eskolemization provides a skolemization method for intuitionistic logic as well. All proofs in [2] were semantical. In this paper a proof-theoretic proof of the completeness of eskolemization with respect to existential quantifiers is presented.
\end{abstract}

Keywords: Skolemization, eskolemization, orderization, Herbrand's theorem, intuitionistic logic, existence logic, Gentzen calculi.

\section{Introduction}

It has been known for a long time that skolemization is not complete for intuitionistic logic. Indeed, the following formulas provide counterexamples showing that the skolemization of these formulas is derivable while the formulas themselves are not.

$$
\begin{array}{ll}
\nvdash_{\mathrm{IQC}} \forall x(A x \vee B) \rightarrow(\forall x A x \vee B) & \vdash_{\mathrm{IQC}} \forall x(A x \vee B) \rightarrow(A c \vee B) \\
\nvdash_{\mathrm{IQC}} \neg \neg \exists x A x \rightarrow \exists x \neg \neg A x & \vdash_{\mathrm{IQC}} \neg \neg A c \rightarrow \exists x \neg \neg A x \\
\nvdash_{\mathrm{IQC}} \forall x \neg \neg A x \rightarrow \neg \neg \forall x A x & \vdash_{\mathrm{IQC}} \forall x \neg \neg A x \rightarrow \neg \neg A c
\end{array}
$$

In [2] the authors introduced an alternative method to replace strong quantifiers by skolem terms that closely resembles skolemization and uses an existence predicate first introduced by Dana Scott in [14]. Under this translation, called eskolemization, negative occurrences of existential quantifiers $\exists x A x$ are replaced by $E t \wedge A t$ and positive occurrences of universal quantifiers $\forall x A x$ by $E t \rightarrow A t$,

\footnotetext{
*Support by the Austrian Science Fund under project P17503 is gratefully acknowledged.
} 
where $E t$ denotes that the term $t$ exists. In [14] Scott presented a natural conservative extension IQCE of intuitionistic logic IQC that captures the notion of existence, and in [1] the authors introduced a Gentzen calculus LJE for this logic satisfying properties like interpolation and cut-elimination. In [2] it was shown that for existential quantifiers eskolemization is sound and complete for existence logic, and satisfies a similar property with respect to IQC. That is, for $A$ not containing the existence predicate,

$$
\vdash_{\mathrm{IQC}} A \Leftrightarrow \vdash_{\mathrm{IQCE}} A \Leftrightarrow \vdash_{\mathrm{IQCE}} A^{e},
$$

where $A^{e}$ denotes the result of eskolemizing the strong existential quantifiers of $A$ (and leaving the strong universal quantifiers unchanged).

In [3] the authors extended this work to universal quantifiers by increasing the expressive power of IQCE via a preorder $\preccurlyeq$, and constructing a skolemization method, called orderization, based on that. Although resembling eskolemization, orderization is less well-behaved in that it introduces new weak quantifiers in a formula. In [3] it has been shown that for $A$ not containing the extra symbols of IQCO,

$$
\vdash_{\mathrm{IQC}} A \Leftrightarrow \vdash_{\mathrm{IQCO}} A \Leftrightarrow \vdash_{\mathrm{IQCO}} A^{o},
$$

where $A^{\circ}$ denotes the orderization of $A$, and IQCO the logic that contains the preorder and the existence predicate.

In this paper we restrict our attention to eskolemization. We do not prove a new result, but present a proof-theoretic proof of the completeness of eskolemization with respect to existential quantifiers. In the original paper [2] this theorem was proved by semantical means, using transformations of Kripke models. This method emerged from the study of the reason for the failure of skolemization in IQC. However, since skolemization is a proof-theoretic property, with numerous applications in computer science, a proof-theoretic proof of the completeness theorem is useful. Although the proof is not difficult, the semantical proof of completeness was found first, and one could wonder whether without this semantical intuition, the eskolemization method would have been discovered at all.

\section{The Gentzen calculus LJE}

In this section we define the Gentzen calculus LJE, which is based on LJ and includes the existence predicate $E$. In the system, $E t$ stands for " $t$ exists". Such a system was first introduced by Scott in [14], but then in a Hilbert style formulation, and called IQCE. The Gentzen calculus for this system was introduced by the authors in [1]. It is in fact based on G3i [19] rather than LJ because it does not contain rules for weakening and contraction.

Our language is denoted by $\mathcal{L}_{e}$. It is a language for predicate logic extended by the symbol $E$, and it contains for every arity infinitely many functions of that arity. Given an existence predicate, terms, including variables, typically range 
over existing as well as non-existing elements, while the quantifiers range over existing objects only.

\section{The calculus LJE}

$$
\begin{aligned}
& A x \Gamma, P \Rightarrow P \quad(P \text { atomic }) \\
& L \perp \Gamma, \perp \Rightarrow C \\
& \mathrm{~L} \wedge \frac{\Gamma, A, B \Rightarrow C}{\Gamma, A \wedge B \Rightarrow C} \\
& \mathrm{R} \wedge \frac{\Gamma \Rightarrow A \quad \Gamma \Rightarrow B}{\Gamma \Rightarrow A \wedge B} \\
& \mathrm{~L} \vee \frac{\Gamma, A \Rightarrow C \quad \Gamma, B \Rightarrow C}{\Gamma, A \vee B \Rightarrow C} \\
& \mathrm{R} \vee \frac{\Gamma \Rightarrow A_{i}}{\Gamma \Rightarrow A_{0} \vee A_{1}} i=0,1 \\
& \mathrm{~L} \rightarrow \frac{\Gamma, A \rightarrow B \Rightarrow A \quad \Gamma, B \Rightarrow C}{\Gamma, A \rightarrow B \Rightarrow C} \\
& \mathrm{R} \rightarrow \frac{\Gamma, A \Rightarrow B}{\Gamma \Rightarrow A \rightarrow B} \\
& \mathrm{~L} \forall \frac{\Gamma, \forall x A x, A t \Rightarrow C \quad \Gamma, \forall x A x \Rightarrow E t}{\Gamma, \forall x A x \Rightarrow C} \\
& \mathrm{R} \forall \frac{\Gamma, E y \Rightarrow A y}{\Gamma \Rightarrow \forall x A[x / y]} * \\
& \mathrm{~L} \exists \frac{\Gamma, A y, E y \Rightarrow C}{\Gamma, \exists x A[x / y] \Rightarrow C} * \\
& \mathrm{R} \exists \frac{\Gamma \Rightarrow A t \quad \Gamma \Rightarrow E t}{\Gamma \Rightarrow \exists x A x} \\
& \operatorname{Cut} \frac{\Gamma \Rightarrow A \quad \Gamma, A \Rightarrow C}{\Gamma \Rightarrow C}
\end{aligned}
$$

Here $(*)$ denotes the condition that the eigen variable $y$ does not occur free in $\Gamma$ and $C$. The term $t$ in $R \exists$ and $L \forall$ is called the main term of the inference. We assume that in a proof bound variables and eigen variables are all different. Proofs are assumed to be trees. We write $\vdash_{\text {LJE }} S$ if the sequent $S$ is derivable in LJE. Given a sequent in a derivation, the depth of the sequent is the maximal length of the branches that lead from a leaf in the derivation to the sequent.

In our main theorem we will need the notion of main formula and ancestor of a formula. The main formulas in the axiom $A x$ are the $P$ 's, and in $L \perp$ it is $\perp$. For all rules for the connectives the formulas $A$ and $B$ are main formulas, as well as $A \wedge B$ in $L \wedge, A \rightarrow B$ in $L \rightarrow$, et cetera. In $L \forall$ and $R \exists$, At is a main formula, and respectively $\forall x A x$ and $\exists x A x$. In $R \forall$ and $L \exists, A y$ is a main formula, and respectively $\forall x A[x / y]$ and $\exists x A[x / y]$. All other formulas in rules are called side formulas.

For every rule, the main formulas in the premises are ancestors of the main formulas of the conclusion. A side formula in the premise is an ancestor of the corresponding side formula in the conclusion. Given a proof, the ancestor relation is the reflexive transitive closure of this relation. A formula is introduced 
along a branch when it is the main formula in the conclusion of a rule along $b$. For example, $\exists x A x$ is introduced both along the branch through the left premise and along the branch through the right premise of $R \exists$.

As an example, in the following proof, the left occurrence of $P$ and the right occurrence of $Q$ in the axiom are ancestors of the formula $P \rightarrow Q$ in the endsequent, and the left occurrence of $Q$ in the top sequent is an ancestor of the left occurrence of $Q$ in the bottom sequent:

$$
\frac{P, Q \Rightarrow Q}{Q \Rightarrow P \rightarrow Q}
$$

\section{The calculus $\mathrm{LJE}_{\mathcal{L}}$}

The calculus LJE contains no axioms stating that certain terms exist. This implies, for example, that $\Rightarrow \exists x E x$ and $\forall x P x \Rightarrow P t$ are not derivable. $\forall x P x, E t \Rightarrow$ $P t$, on the other hand, is derivable in the system. As we will see, the crucial ingredient in the completeness proof of eskolemization is that all terms in a sequent $S$ exist, while the skolem terms in $S^{e}$ do not. Therefore we isolate a language $\mathcal{L}$ in $\mathcal{L}_{e}$ that contains at least one constant, all predicates of $\mathcal{L}$, and such that $\mathcal{L}_{e} \backslash \mathcal{L}$ still contains infinitely many functions of every arity. The original sequents will be in $\mathcal{L}$, while the eskolemized sequents will contain terms that belong to $\mathcal{L}_{e} \backslash \mathcal{L}$. Let $T_{\mathcal{L}}$ denote the set of closed terms in $\mathcal{L}$. It thus is our aim to build a system in which all terms in $T_{\mathcal{L}}$ exist, and we therefore define

$$
A x_{\mathcal{L}} \equiv_{\text {def }}\left\{\Gamma \Rightarrow E t \mid t \in \mathcal{T}_{\mathcal{L}}, \Gamma \text { a multiset }\right\} .
$$

Note that because of the assumptions on $\mathcal{L}, A x_{\mathcal{L}}$ contains at least one sequent. $\mathrm{LJE}_{\mathcal{L}}$ denotes the system LJE extended by the axioms $A x_{\mathcal{L}}$. We sometimes write $\vdash$ for $\vdash_{\mathrm{LJE}}$, and $\vdash_{\mathcal{L}}$ for $\vdash_{\mathrm{LJE}_{\mathcal{L}}}$, the $\mathcal{L}$ indicating that we assume the terms in $\mathcal{L}$ to exist.

\section{Example 1}

$$
\begin{gathered}
\forall \Rightarrow \exists x E x \quad \forall \Rightarrow \forall x E x . \\
\vdash_{\mathcal{L}} \Rightarrow \exists x E x \wedge \forall x E x .
\end{gathered}
$$

Observe that given another predicate $E^{\prime}$ that satisfies the same rules of LJE as $E$, it follows that

$$
\vdash_{\mathcal{L}} E t \Rightarrow E^{\prime} t \text { and } \vdash_{\mathcal{L}} E^{\prime} t \Rightarrow E t
$$

We namely have that $\vdash_{\mathcal{L}}\left(\Rightarrow \forall x E x \wedge \forall x E^{\prime} x\right)$, and also $\vdash_{\mathcal{L}}\left(\forall x E x, E^{\prime} t \Rightarrow E t\right)$ and $\vdash_{\mathcal{L}}\left(\forall x E^{\prime} x, E t \Rightarrow E^{\prime} t\right)$. Finally, two cuts do the trick. This shows that the existence predicate $E$ is unique up to provable equivalence.

It is easy to see that the following lemma holds.

Proposition 1 [1] For all sequents $S$ in $\mathcal{L}$ :

$$
\vdash_{\text {LJ }} S \text { if and only if } \vdash_{\mathcal{L}} S \text {. }
$$




\section{Properties of LJE}

In this section we recall some results from [1] that show that LJE and $\mathrm{LJE}_{\mathcal{L}}$ are well-behaved proof systems.

Theorem 1 [1] (ECut theorem)

For $\mathrm{L} \in\left\{\mathrm{LJE}, \mathrm{LJ} \mathrm{E}_{\mathcal{L}}\right\}$ : Every sequent in $\mathcal{L}_{e}$ provable in $\mathrm{L}$ has a proof in $\mathrm{L}$ in which the only cuts are instances of the Ecut rule:

$$
\frac{\Gamma \Rightarrow E t \quad \Gamma, E t \Rightarrow C}{\Gamma \Rightarrow C} \text { Ecut }
$$

where $\Gamma \Rightarrow E t$ is in $A x_{\mathcal{L}}$. Such proofs are called ecut-free. In particular, LJE has cut-elimination.

Corollary $1 \mathrm{LJE}_{\mathcal{L}}$ and LJE are consistent.

Corollary 2 For quantifier free closed sequents the relations $\vdash$ and $\vdash_{\mathcal{L}}$ are decidable.

Corollary 3 [1] LJE and $\mathrm{LJE}_{\mathcal{L}}$ have interpolation and satisfy the Beth definability property.

We write $\vdash_{\mathcal{L}}^{c} S$ when $A$ has an ecut-free proof in $\operatorname{LJE}_{\mathcal{L}}$. The following lemma follows almost immediately from the cut-elimination theorem. We state it explicitly because we will use it several times in what follows.

\section{Lemma 1}

$$
\begin{array}{ll}
\vdash_{\mathcal{L}}^{c} \Gamma \Rightarrow C & \Rightarrow \vdash_{\mathcal{L}}^{c} \Gamma, A \Rightarrow C \\
\vdash_{\mathcal{L}}^{c} \Gamma, A \wedge B \Rightarrow C & \Rightarrow \vdash_{\mathcal{L}}^{c} \Gamma, A, B \Rightarrow C \\
\vdash_{\mathcal{L}}^{c} \Gamma, A \vee B \Rightarrow C & \Rightarrow \vdash_{\mathcal{L}}^{c} \Gamma, A \Rightarrow C \text { and } \vdash_{\mathcal{L}}^{c} \Gamma, B \Rightarrow C \\
\vdash_{\mathcal{L}}^{c} \Gamma, A \rightarrow B \Rightarrow C & \Rightarrow \vdash_{\mathcal{L}}^{c} \Gamma, B \Rightarrow C \\
\vdash_{\mathcal{L}}^{c} \Gamma, \exists x A x \Rightarrow C & \Rightarrow \vdash_{\mathcal{L}}^{c} \Gamma, E x, A x \Rightarrow C(x \text { not free in } \Gamma, C)
\end{array}
$$

\section{Eskolemization}

In this section we recall the eskolemization procedure first introduced in [2]. $Q$ denotes either $\forall$ or $\exists$. A strong quantifier in a formula $A$ is the occurrence of a subformula of the form $Q x B(x)$ in $A$, where $Q=\forall$ if the occurrence is positive, and $Q=\exists$ if the occurrence is negative. The first strong quantifier in $A$ is the first strong quantifier occurrence in $A$ when reading $A$ from left to right.

The eskolem sequence of a formula $A$ is a sequence of formulas $A=A_{1}, \ldots, A_{n}=$ $A^{E}$ such that $A_{n}$ does not contain any strong quantifiers and $A_{i+1}$ is the result of replacing the first strong quantifier $Q x B(x)$ in $A_{i}$ by

$$
E f\left(y_{1}, \ldots, y_{n}\right) \rightarrow B\left(f\left(y_{1}, \ldots, y_{n}\right)\right) \text { if } Q=\forall
$$


and by

$$
E f\left(y_{1}, \ldots, y_{n}\right) \wedge B\left(f\left(y_{1}, \ldots, y_{n}\right)\right) \text { if } Q=\exists,
$$

where $f \in \mathcal{L}_{e} \backslash \mathcal{L}$ does no occur in $A_{i}$, and the weak quantifiers in the scope of which $Q x B(x)$ occurs are exactly $Q_{1} y_{1}, \ldots, Q_{n} y_{n}$. This definition is carried over to sequents by treating a sequent $\Gamma \Rightarrow \Delta$ as the formula $\wedge \Gamma \rightarrow \bigvee \Delta$.

This transformation is called existence skolemization. If it is carried out only for the strong existential quantifiers in $S$ we denote the result by $S^{e}$, and call the procedure eskolemization, and $S^{e}$ the eskolemization of $S$.

Note that if $Q x B(x)$ is not in the scope of weak quantifiers, then $f$ is a constant. Also note that in eskolemization occurrences of formulas are replaced rather than formulas. For example, if $S$ is the sequent $\exists x B x \wedge \exists x B x \Rightarrow$, then $S^{e}$ is $E c \wedge B c \wedge E d \wedge B d \Rightarrow$ and not $E c \wedge B c \wedge E c \wedge B c \Rightarrow$. Note that given $S, S^{e}$ is unique up to renaming of the skolem functions. Therefore we speak of the eskolemization of a sequent.

Observe that classical skolemization is existence skolemization without the existence predicate, that is, without " $E f\left(y_{1}, \ldots, y_{n}\right) \rightarrow$ " and " $E f\left(y_{1}, \ldots, y_{n}\right) \wedge$ ". Clearly, $\vdash$ LJE $A \Rightarrow A^{e}$. Hence also

$$
\vdash_{\mathcal{L}} S \Rightarrow \vdash_{\mathcal{L}} S^{e}
$$

Here follow some examples of eskolemization, where $Q$ and $P$ are unary predicates, and $c$ and $f$ are in the skolem language $\mathcal{L}_{e} \backslash \mathcal{L}$.

$$
\begin{array}{cc}
S & S^{e} \\
\exists x P(x) \Rightarrow \forall x Q(x) & E c \wedge P(c) \Rightarrow \forall x Q(x) \\
\forall x \exists y R(x, y) \Rightarrow & \forall x(E f x \wedge R(x, f x)) \Rightarrow
\end{array}
$$

It is shown in [2] that

$$
\begin{array}{ll}
\forall \text { LJE } \forall x(A x \vee B) \Rightarrow(\forall x A x \vee B) & \forall \text { LJE } \forall x(A x \vee B) \Rightarrow((E c \rightarrow A c) \vee B) \\
\forall \text { LJE } \neg \neg \exists x A x \rightarrow \exists x \neg \neg A x & \forall \text { LJE } \neg \neg(E c \wedge A c) \rightarrow \exists x \neg \neg A x .
\end{array}
$$

Thus although these sequents are counterexamples to the completeness of skolemization, since IQC derives $\forall x(A x \vee B) \Rightarrow(A c \vee B)$ and $\neg \neg A c \rightarrow \exists x \neg \neg A x$, they no longer are for eskolemization.

That eskolemization is not complete with respect to universal quantifiers shows the following example:

$$
\forall \text { IQC } \forall x \neg \neg A x \rightarrow \neg \neg \forall x A x \quad \vdash_{\text {IQCE }} \forall x \neg \neg A x \rightarrow \neg \neg(E c \rightarrow A c) .
$$

As explained in the introduction, in [3] an alternative skolemization method is developed that is sound and complete with respect to both existential and universal quantifiers. 


\section{$5 \quad$ Proof idea}

In this section the idea behind the completeness proof of eskolemization will be explained, and some technical notions will be introduced. Be aware that most definitions concern occurrences of formulas rather than formulas. For example, when considering an occurrence of a formula $A$ in a sequent $S$ in a proof, then saying that $A$ occurs in another sequent $S^{\prime}$ somewhere in the proof above $S$, means that a formula $A$ which is an ancestor of the particular occurrence of $A$ in $S$ that we consider, occurs in $S^{\prime}$. If it is clear from the context that occurrences of formulas are considered rather than formulas, the word "occurrence" will be omitted.

\subsection{Definition of $S^{B}$}

Consider a derivation $\mathcal{D}$ and a formula $A t \wedge E t$ that occurs in the endsequent of $\mathcal{D}$. For any sequent $S$ in $\mathcal{D}$ and any formula $B, S^{B}$ denotes the result of replacing any occurrence of $A t \wedge E t$ that is an ancestor of the same formula in the endsequent, by $B$. At $\wedge E t$ is not indicated in $S^{B}$ since it will always be clear from the context which formula will be replaced. Also, we have left out the brackets, but $A t \wedge E t$ should be read as $(A t \wedge E t)$. Note that a formula At $\wedge E t$ might occur at several places in the endsequent, but here it is meant that we have a particular occurrence of the formula in mind, and only replace ancestors of this occurrence.

Observe that if $\exists x_{1} A x_{1}, \ldots, \exists x_{n} A_{n} x_{n}$ are the negative occurrences of existential quantifiers in $S$ and $\exists x_{i} A_{i}$ is not a subformula of $\exists x_{j} A_{j}$ for $j<i$, then

$$
S=\left(\ldots\left(\left(S^{e}\right)^{\exists x_{1} A x_{1}}\right) \ldots\right)^{\exists x_{n} A_{n} x_{n}},
$$

and the formulas $E t_{j} \wedge A t_{j}\left(t_{j}\right.$ being the skolem term of $A_{j}$ in $\left.S^{e}\right)$ occur exactly once in $\left(\ldots\left(\left(S^{e}\right)^{\exists x_{1} A x_{1}}\right) \ldots\right)^{\exists x_{i} A_{n} x_{i}}$ for $j>i$. Note also that the $t_{j}$ are not in $\mathcal{L}$ by the definition of eskolemization. In the completeness theorem we thus have to show that the derivability of $S^{e}$ implies the derivability of $\left(\ldots\left(\left(S^{e}\right)^{\exists x_{1} A x_{1}}\right) \ldots\right)^{\exists x_{n} A_{n} x_{n}}$. We will do so by proving that given a negative and unique occurrence of $A t \wedge E t$ in $S$, where $t \notin \mathcal{L}$, the derivability of $S$ implies the derivability of $S^{\exists x A x}$.

The proof of this fact will consist of several transformations on ecut-free proofs that turn a proof of $S$ into a proof of $S^{\exists x A x}$. It is based on the naive idea to insert an application of $L \exists$ to $A t, E t$ below the sequent in which $A t$ and $E t$ appear as elements for the last time along a branch, as in this example:

$$
\frac{A t, E t \Rightarrow B \quad A t, E t \Rightarrow C}{\frac{A t, E t \Rightarrow B \wedge C}{A t \wedge E t \Rightarrow B \wedge C}}
$$

Indeed, the following is a valid proof of $\exists x A x \Rightarrow B \wedge C$, provided that $t$ does 
not occur in $B$ and $C$ :

$$
\frac{A t, E t \Rightarrow B \quad A t, E t \Rightarrow C}{\frac{A t, E t \Rightarrow B \wedge C}{\exists x A x \Rightarrow B \wedge C}}
$$

There are two reasons why this strategy might not work: it might be that there are no sequents of which $A t$ and $E t$ are elements, or there are, but the eigen variable condition on $t$ might block the application of $L \exists$ to At, Et. The first case is easily dealt with, but the second problem requires several transformations on proofs. For the latter, recall that $A t \wedge E t$ has a unique occurrence in $S$ and $t \notin \mathcal{L}$. Consider a branch $b$ and the lowest sequent $\Gamma, A t, E t \Rightarrow B$ along it in which $A t$ and $E t$ appear as elements, and suppose $t$ occurs in $\Gamma$ or $B$, say in a formula $C t$. Then either $C t$ has to be an ancestor of $A t \wedge E t$, or it is an ancestor of a formula to which a quantifier rule is applied with main term $t$, because $C t$, containing a term not in $\mathcal{L}$, cannot be a cut formula. Here follow three examples that illustrate these three cases.

Example 2 In the following $A t \wedge E t$ is nowhere introduced:

$$
\frac{\frac{\forall y(A t \wedge E t \wedge B y), A t \wedge E t, B t \Rightarrow B t}{\forall y(A t \wedge E t \wedge B y), A t \wedge E t \wedge B t \Rightarrow B t}}{\forall y(A t \wedge E t \wedge B y) \Rightarrow B t}
$$

Clearly, the following is a valid proof of $\forall y(\exists x A x \wedge B y) \Rightarrow B t$ :

$$
\frac{\frac{\forall y(\exists x A x \wedge B y), \exists x A x, B t \Rightarrow B t}{\forall y(\exists x A x \wedge B y), \exists x A x \wedge B t \Rightarrow B t}}{\forall y(\exists x A x \wedge B y) \Rightarrow B t}
$$

Example 3 Consider the following part of a proof, where consecutive applications of $L \wedge$ are collapsed, and where $t$ only occurs in $A t \wedge E t$ and $C t \vee D t$ :

$$
\frac{\frac{A t, E t, B \Rightarrow C t}{A t \wedge E t \wedge B \Rightarrow C t} \quad \frac{A t, E t, B \Rightarrow E t}{A t \wedge E t \wedge B \Rightarrow C t \vee D t} \quad \frac{A t \wedge E t \wedge B \Rightarrow E t}{A t \wedge E t \wedge B \Rightarrow \exists y(C y \vee D y)}}{L^{A}} R
$$

To obtain a proof of $\exists x A x \wedge B \Rightarrow \exists y(C y \vee D y)$, we cannot just push the application of $R \exists$ up along the left branch, since we move to $A t \wedge E t \wedge B \Rightarrow C t$. However, at the left branch we could mimic the application of rules at the right branch: since the latter have to be left rules, in this case $L \wedge$, we can apply them backwards at the left branch, moving along derivable sequents till $A t, E t$ belongs to the antecedent, while keeping $\exists y(C y \vee D y)$ as the succedent. The result ends as follows, again collapsing consecutive applications of $L \wedge$ :

$$
\frac{A t, E t, B \Rightarrow C t \vee D t \quad A t, E t, B \Rightarrow E t}{\frac{A t, E t, B \Rightarrow \exists y(C y \vee D y)}{A t \wedge E t \wedge B \Rightarrow \exists y(C y \vee D y)}}
$$


Now we can apply $L \exists$ to $A t, E t, B \Rightarrow \exists y(C y \vee D y)$ to obtain a valid proof:

$$
\begin{gathered}
\frac{A t, E t, B \Rightarrow C t \vee D t \quad A t, E t, B \Rightarrow E t}{A t, E t, B \Rightarrow \exists y(C y \vee D y)} \\
\frac{\exists x A x, B \Rightarrow \exists y(C y \vee D y)}{\exists x A x \wedge B \Rightarrow \exists y(C y \vee D y)}
\end{gathered}
$$

Example 4 This example describes the situation that along a branch the sequent $\Gamma, A t, E t \Rightarrow B$ is the last one where $A t, E t$ appear as elements, and there is no quantifier rule with main variable $t$ below it, and where $B$ contains $t$. Since $A t, E t$ do not appear as elements below $\Gamma, A t, E t \Rightarrow B$ this implies that in $B, t$ only occurs in formulas of the form $A t \wedge E t$, as in this example, where for $B$ we have taken the formula $A t \wedge E t \rightarrow B$ :

$$
\frac{\frac{A t, E t \Rightarrow A t \wedge E t \rightarrow B}{A t \wedge E t \Rightarrow A t \wedge E t \rightarrow B} \quad \frac{A t, E t, C \Rightarrow D}{A t \wedge E t, C \Rightarrow D}}{A t \wedge E t,(A t \wedge E t \rightarrow B) \rightarrow C \Rightarrow D}
$$

Suppose $t$ does not belong to $B, C$ and $D$. This is not essential, it just simplifies the example. Here the transformation of proofs is indirect, and makes use of the following observations.

First, observe that the derivability of $A t, E t \Rightarrow A t \wedge E t \rightarrow B$ implies the derivability of $A t, E t \Rightarrow \top \rightarrow B$. Thus the following is a valid derivation:

$$
\frac{\frac{A t, E t \Rightarrow \top \rightarrow B}{A t \wedge E t \Rightarrow \top \rightarrow B} \quad \frac{A t, E t, C \Rightarrow D}{A t \wedge E t, C \Rightarrow D}}{A t \wedge E t,(\top \rightarrow B) \rightarrow C \Rightarrow D}
$$

Because we have removed $t$ in the top sequents except in $A t, E t$, we can now safely apply $L \exists$ to them and obtain

$$
\frac{\frac{A t, E t \Rightarrow \top \rightarrow B}{\exists x A x \Rightarrow \top \rightarrow B} \quad \frac{A t, E t, C \Rightarrow D}{\exists x A x, C \Rightarrow D}}{\exists x A x,(\top \rightarrow B) \rightarrow C \Rightarrow D}
$$

Since $A t, E t$ or $\exists x A x$ is an element of the antecedents of these sequents, replacing $\top$ by $\exists x A x$ results in the following valid derivation:

$$
\begin{gathered}
\frac{A t, E t \Rightarrow \exists x A x \rightarrow B}{\exists x A x \Rightarrow \exists x A x \rightarrow B} \quad \frac{A t, E t, C \Rightarrow D}{\exists x A x, C \Rightarrow D} \\
\exists x A x,(\exists x A x \rightarrow B) \rightarrow C \Rightarrow D
\end{gathered}
$$

These three examples illustrate the main ideas in the completeness proof. We proceed with the technical details. 


\subsection{Definition of $S_{b}$}

We remind the reader that in what follows, if we consider an occurrence of a formula $A t \wedge E t$ in the endsequent of a proof, when we speak about formulas $A t, E t$ or $A t \wedge E t$ in the proof we always mean ancestors of that particular occurrence of $A t \wedge E t$ in the endsequent, even if we do not explicitly say so.

Given a proof $\mathcal{D}$ of $S$ and a branch $b$ in it, we distinguish three cases:

1. At $\wedge E t$ is introduced along $b$ and below it there is an application of a quantifier rule with main term $t$. The conclusion of the lowest such rule is denoted by $S_{b}^{\mathcal{D}}$, and the depth of the right hypothesis of this rule by $t_{b}$. Example 3 illustrates this situation.

2. At $\wedge E t$ is introduced along $b$ and there is no application of a quantifier rule with main term $t$ below the last introduction of $A t \wedge E t$ along $b$. Then $S_{b}$ denotes the hypothesis of the rule where $A t \wedge E t$ is introduced for the last time along $b$ and $S_{b}^{\prime}$ its conclusion, and $t_{b}=0$. Example 4 illustrates this situation.

3. At $\wedge E t$ is nowhere introduced along $b$. Then $t_{b}=0$ and $S_{b}$ is the leaf of $b$. Example 2 illustrates this situation.

When $\mathcal{D}$ is clear from the context we omit the superscript. We do not indicate $A t \wedge E t$ in the notation, since it will always be clear from the context with respect to which formula of the form $A t \wedge E t$ the $t_{b}$ and $S_{b}$ have to be taken.

Note that in the first case the quantifier rules cannot be $L \exists$ or $R \forall$ because of the fact that its hypotheses contain $A t \wedge E t$, which would violate the eigen variable condition. Thus they are $R \exists$ or $L \forall$, and therefore indeed have right hypotheses.

In Examples 3 and 4 , let $b$ be the left branch and $c$ the right one. In Example 3 $S_{b}=S_{c}=A t \wedge E t \wedge B \Rightarrow \exists y(C y \vee D y)$, and $t_{b}=t_{c}$ is the depth of $A t \wedge E t \wedge B \Rightarrow$ $E t$. In Example $4 S_{b}=A t, E t \Rightarrow A t \wedge E t \rightarrow B$ and $S_{b}^{\prime}=A t \wedge E t \Rightarrow A t \wedge E t \rightarrow B$, and $S_{c}=A t, E t, C \Rightarrow D$ and $S_{c}^{\prime}=A t \wedge E t, C \Rightarrow D$ and $t_{b}=t_{c}=0$ since there are no quantifier rules with main term $t$ below the last introduction of $A t \wedge E t$ along these branches. In Example $2 S_{b}$ is the top sequent and $t_{b}=0$.

Remark 1 Note that although we do not write brackets in some formulas, they are there. For example, if $B=C \wedge D, A \wedge B$ stands for $(A \wedge(C \wedge D))$, so that in can never be the case that $A \wedge B$ is introduced while $A$ and $B$ are not the main formulas. In what follows, $A t \wedge E t$ always stands for $(A t \wedge E t)$. Therefore, if $A t \wedge E t$ is introduced, it is so via an application of $L \wedge$ to $A t$ and $E t$. Therefore in case $2 ., S_{b}$ contains $A t$ and $E t$ in the antecedent.

\subsection{An order}

In the completeness theorem we need the following order on finite multisets of natural numbers. Given a multiset $I$ and natural number $n, I_{n}$ denotes the 
number of occurrences of $n$ in $I$. We define the following ordering:

$$
I \prec J \equiv_{\text {def }} \forall n \in \mathbb{N}: I_{n}<J_{n} \text { or } \exists m>n J_{m} \neq 0 .
$$

It is not difficult to see that on finite multisets of natural numbers $\prec$ is a wellfounded linear order.

With every derivation $\mathcal{D}$ in which endsequent occurs a formula $A t \wedge E t$, we associate a multiset of natural numbers:

$$
\mathcal{D}^{t} \equiv_{\text {def }}\left\{t_{b} \mid b \text { is a branch in } \mathcal{D}\right\}
$$

and call it the order of $\mathcal{D}$.

\section{Completeness}

The main part of the completeness proof for eskolemization consists of the following technical lemma. Recall that when speaking about formulas, most of the time we mean occurrences of formulas, even if we do not explicitly say so.

Lemma 2 If a sequent $S$ contains exactly one occurrence of a formula $A t \wedge E t$, $A t \wedge E t$ occurs negatively and $t$ occurs in $S$ only in this formula, then if $S$ has an ecut-free proof, there exists an ecut-free proof $\mathcal{D}$ of $S$ for which $\mathcal{D}^{t}$ consists of zeros only.

Proof It suffices to show that for any ecut-free proof $\mathcal{D}$ of $S$ as in the lemma, if it contains a branch $b$ for which $t_{b}>0$, there is an ecut-free proof $\mathcal{D}^{\prime}$ of $S$ for which $\left(\mathcal{D}^{\prime}\right)^{t} \prec \mathcal{D}^{t}$. Therefore consider such an ecut-free proof $\mathcal{D}$ with a branch $b$ for which $t_{b}>0$, and choose $b$ such that it is maximal in $\mathcal{D}^{t}$. There might, of course, be more than one such branch; we just pick an arbitrary one.

As observed above, $S_{b}$ is the conclusion of $R \exists$ or $L \forall$. We first treat the existential quantifier (I), and then the universal one (II).

(I) In this case the subproof $\mathcal{D}_{b}$ of $S_{b}=\Gamma \Rightarrow \exists y D y$ is

$$
\frac{\Gamma \Rightarrow D t \quad \frac{\mathcal{D}_{e}}{\Gamma \Rightarrow E t}}{\Gamma \Rightarrow \exists y D y}
$$

Because $t_{b}>0, \Gamma \Rightarrow E t$ is not an axiom. We distinguish by cases according to the last rule of $\mathcal{D}_{e}$. For most cases the main idea is the same, but the details vary from case to case, and we therefore treat them all.

Suppose the last rule of $\mathcal{D}_{e}$ is $L \vee$. Then $S_{b}=\Pi, B \vee C \Rightarrow \exists y D y$ and $\mathcal{D}_{b}$ ends as follows:

$$
\frac{\Pi, B \vee C \Rightarrow D t}{\Pi, \frac{\Pi, B \Rightarrow E t \quad \Pi, C \Rightarrow E t}{\Pi, B \vee C \Rightarrow E t}}
$$


By Lemma 1 the sequents $\Pi, B \Rightarrow D t$ and $\Pi, C \Rightarrow D t$ have ecut-free proofs. Therefore there exists an ecut-free proof $\mathcal{D}_{b}^{\prime}$ of $S_{b}$ that ends as follows:

$$
\begin{gathered}
\frac{\Pi, B \Rightarrow D t \quad \Pi, B \Rightarrow E t}{\frac{\Pi, B \Rightarrow \exists y D y}{\Pi, B \vee C \Rightarrow \exists y D y}} \quad \frac{\Pi, C \Rightarrow D t \quad \Pi, C \Rightarrow E t}{\Pi, C \Rightarrow \exists y D y} \\
\hline
\end{gathered}
$$

Let $\mathcal{D}^{\prime}$ be the result of replacing $\mathcal{D}_{b}$ in $\mathcal{D}$ by $\mathcal{D}_{b}^{\prime}$. We show that $\left(\mathcal{D}^{\prime}\right)^{t} \prec \mathcal{D}^{t}$. Let $\mathcal{B}$ be the set of branches in $\mathcal{D}$ through $\Pi, B \vee C \Rightarrow \exists y D y$, and $\mathcal{B}^{\prime}$ the set of branches in $\mathcal{D}^{\prime}$ through $\Pi, B \vee C \Rightarrow \exists y D y$. It suffices to show that for all branches $c$ in $\mathcal{B}^{\prime}, t_{c}<t_{b}$. If $t_{c}=0$, it is immediate, since $t_{b}>0$. If $t_{c}>0$, then $A t \wedge E t$ is introduced along $c$, and that has to happen above $S_{b}$ because by definition this does not happen below it. Hence $S_{c}$ is $\Pi, B \Rightarrow \exists y D y$ or $\Pi, C \Rightarrow \exists y D y$, and $t_{c}$ is the depth of the subproof of $\Pi, B \Rightarrow E t$ or $\Pi, C \Rightarrow E t$. Thus $t_{c}$ is smaller than the depth of $\mathcal{D}_{e}$, whence $t_{c}<t_{b}$. This completes the case $L \vee$.

Suppose the last rule of $\mathcal{D}_{e}$ is $L \wedge$. Then $S_{b}=\Pi, B \wedge C \Rightarrow \exists y D y$ and $\mathcal{D}_{b}$ ends as follows:

$$
\frac{\Pi, B \wedge C \Rightarrow D t \quad \frac{\Gamma, B, C \Rightarrow E t}{\Pi, B \wedge C \Rightarrow E t}}{\Pi, B \wedge C \Rightarrow \exists y D y}
$$

By Lemma 1 the sequent $\Pi, B, C \Rightarrow D t$ has an ecut-free proof and we thus have an ecut-free proof $\mathcal{D}_{b}^{\prime}$ of $S_{b}$ that ends as follows:

$$
\frac{\Gamma, B, C \Rightarrow D t \quad \Gamma, B, C \Rightarrow E t}{\Pi, B, C \Rightarrow \exists y D y}
$$

Let $\mathcal{D}^{\prime}$ be the result of replacing $\mathcal{D}_{b}$ in $\mathcal{D}$ by $\mathcal{D}_{b}^{\prime}$. We show that $\left(\mathcal{D}^{\prime}\right)^{t} \prec \mathcal{D}^{t}$. Let $\mathcal{B}$ be the set of branches in $\mathcal{D}$ through $\Pi, B \wedge C \Rightarrow \exists y D y$ in $\mathcal{D}$ and $\mathcal{B}^{\prime}$ the set of branches through $\Pi, B \wedge C \Rightarrow \exists y D y$ in $\mathcal{D}^{\prime}$. It suffices to show that for all branches $c$ in $\mathcal{B}^{\prime}$ we have $t_{c}<t_{b}$. Suppose $c \in \mathcal{B}^{\prime}$. If $t_{c}=0, t_{c}<t_{b}$ since $t_{b}>0$. If $t_{c}>0$, then $A t \wedge E t$ is introduced along $c$. If this happens for the last time above $S_{b}$, clearly $t_{c}<t_{b}$. If it does not, the last application of $\mathcal{D}_{e}$, and whence of $\left(\mathcal{D}^{\prime}\right)_{b}$, has to be an application of $L \wedge$ to $B=A t$ and $C=E t$. Hence there is no quantifier rule with main variable $t$ below it, and thus $t_{c}=0$. This completes the case $L \wedge$.

Suppose the last rule of $\mathcal{D}_{e}$ is $L \rightarrow$. Then $S_{b}=\Pi, B \rightarrow C \Rightarrow \exists y D y$ and and $\mathcal{D}_{b}$ ends as follows:

$$
\frac{\Pi, B \rightarrow C \Rightarrow D t \quad \frac{\Pi, B \rightarrow C \Rightarrow B \quad \Pi, C \Rightarrow E t}{\Pi, B \rightarrow C \Rightarrow E t}}{\Pi, B \rightarrow C \Rightarrow \exists y D y}
$$


By Lemma 1 the sequent $\Pi, C \Rightarrow D t$ has an ecut-free proof and we thus have an ecut-free proof $\mathcal{D}_{b}^{\prime}$ of $S_{b}$ that ends as follows:

$$
\frac{\Pi, B \rightarrow C \Rightarrow B \quad \frac{\Pi, C \Rightarrow D t \quad \Pi, C \Rightarrow E t}{\Pi, C \Rightarrow \exists y D y}}{\Pi, B \rightarrow C \Rightarrow \exists y D y}
$$

Let $\mathcal{D}^{\prime}$ be the result of replacing $\mathcal{D}_{b}$ in $\mathcal{D}$ by $\mathcal{D}_{b}^{\prime}$. The proof that $\left(\mathcal{D}^{\prime}\right)^{t} \prec \mathcal{D}^{t}$ is similar to the cases above.

Suppose the last rule of $\mathcal{D}_{e}$ is $L \forall$. Then $S_{b}=\Pi, \forall z B z \Rightarrow \exists y D y$ and $\mathcal{D}_{b}$ ends as follows:

$$
\frac{\Pi, \forall z B z \Rightarrow D t \quad \frac{\Pi, \forall z B z, B s \Rightarrow E t \quad \Pi, \forall z B z \Rightarrow E s}{\Pi, \forall z B z \Rightarrow E t}}{\Pi, \forall z B z \Rightarrow \exists y D y}
$$

By Lemma 1 the sequent $\Pi, \forall z B z, B s \Rightarrow D t$ has an ecut-free proof, and thus we have an ecut-free proof $\mathcal{D}_{b}^{\prime}$ of $S_{b}$ that ends as follows:

$$
\frac{\Pi, \forall z B z, B s \Rightarrow D t \quad \Pi, \forall z B z, B s \Rightarrow E t}{\frac{\Pi, \forall z B z, B s \Rightarrow \exists y D y}{\Pi, \forall z B z \Rightarrow \exists y D y} \Pi, \forall z B z \Rightarrow E s}
$$

If $s \neq t$, let $\mathcal{D}^{\prime}$ be the result of replacing $\mathcal{D}_{b}$ in $\mathcal{D}$ by $\mathcal{D}_{b}^{\prime}$. The argument showing that $\left(\mathcal{D}^{\prime}\right)^{t} \prec \mathcal{D}^{t}$ is similar to the cases above. If $s=t$, the sequent $\Pi, \forall z B z \Rightarrow E s$ equals $\Pi, \forall z B z \Rightarrow E t$, where the former has lower depth than the latter. Therefore we let $\mathcal{D}^{\prime}$ be the result of replacing $\mathcal{D}_{e}$ by the derivation of this $\Pi, \forall z B z \Rightarrow E s$, and apply the same reasoning as before.

Suppose the last rule of $\mathcal{D}_{e}$ is $L \exists$. Then $S_{b}=(\Pi, \exists x B x \Rightarrow E t)$ and $\mathcal{D}_{b}$ ends as follows:

$$
\frac{\Pi, \exists x B x \Rightarrow D t \quad \frac{\Pi, E x, B x \Rightarrow E t}{\Pi, \exists x B x \Rightarrow E t}}{\Pi, \exists x B x \Rightarrow \exists y D y}
$$

where $x$ is not free in $\Pi$ and $t$. Pick a $z$ that does not occur in $\mathcal{D}_{b}$. By Lemma 1 there exists an ecut-free proof of $\Pi, E z, B z \Rightarrow D t$. Let $\mathcal{D}^{\prime}$ be the result of replacing $\mathcal{D}_{b}$ in $\mathcal{D}$ by $\mathcal{D}_{b}^{\prime}$, which is

$$
\frac{\Pi, E z, B z \Rightarrow D t \quad \Pi, E z, B z \Rightarrow E t}{\Pi, E z, B z \Rightarrow \exists y D y}
$$

That $\mathcal{D}^{\prime}$ is an ecut-free proof and $\left(\mathcal{D}^{\prime}\right)^{t} \prec \mathcal{D}^{t}$ is shown in a similar way as in the other cases.

Suppose the last rule of $\mathcal{D}_{e}$ is an Ecut:

$$
\frac{\Gamma \Rightarrow D t \quad \frac{\Gamma \Rightarrow E s \quad \Gamma, E s \Rightarrow E t}{\Gamma \Rightarrow E t}}{\Gamma \Rightarrow \exists y D y}
$$


where $\Gamma \Rightarrow E s$ is an axiom in $A x_{\mathcal{L}}$. By Lemma 1 there exists an ecut-free proof of $E s, \Gamma \Rightarrow D t$. We replace $\mathcal{D}_{b}$ in $\mathcal{D}$ by the following derivation:

$$
\frac{\Gamma \Rightarrow E s \quad \frac{E s, \Gamma \Rightarrow D t \quad E s, \Gamma \Rightarrow E t}{E s, \Gamma \Rightarrow \exists y D y}}{\Gamma \Rightarrow \exists y D y}
$$

The result, $\mathcal{D}^{\prime}$ is a derivation for which $\left(\mathcal{D}^{\prime}\right)^{t} \prec \mathcal{D}^{t}$. This completes case (I).

(II) We turn to the case that $S_{b}$ is the conclusion of $L \forall$, which is treated in a similar way. In this case the subproof $\mathcal{D}_{b}$ of $S_{b}=\Gamma, \forall y D y \Rightarrow F$ is

$$
\frac{\Gamma, \forall y D y, D t \Rightarrow F \quad \frac{\mathcal{D}_{e}}{\Gamma, \forall y D y \Rightarrow E t}}{\Gamma, \forall y D y \Rightarrow F}
$$

Because $t_{b}>0, \Gamma, \forall y D y \Rightarrow E t$ is not an axiom. We distinguish by cases according to the last rule of $\mathcal{D}_{e}$. All cases except $L \forall$ are treated in a similar way as above. As an example, we treat $L \wedge$ and $L \forall$.

Suppose the last rule of $\mathcal{D}_{e}$ is $L \wedge$. Then $S_{b}=\Pi, \forall y D y, B \wedge C \Rightarrow F$ and $\mathcal{D}_{b}$ ends as follows:

$$
\frac{\Pi, \forall y D y, D t, B \wedge C \Rightarrow F \quad \frac{\Pi, \forall y D y, B, C \Rightarrow E t}{\Pi, \forall y D y, B \wedge C \Rightarrow E t}}{\Pi, \forall y D y, B \wedge C \Rightarrow F}
$$

By Lemma 1 the sequent $\Pi, \forall y D y, B, C \Rightarrow F$ has an ecut-free proof. Therefore there exists an ecut-free proof $\mathcal{D}_{b}^{\prime}$ of $S_{b}$ that ends as follows:

$$
\frac{\Pi, \forall y D y, D t, B, C \Rightarrow F \quad \Pi, \forall y D y, B, C \Rightarrow E t}{\frac{\Pi, \forall y D y, B, C \Rightarrow F}{\Pi, \forall y D y, B \wedge C \Rightarrow F}}
$$

Let $\mathcal{D}^{\prime}$ be the result of replacing $\mathcal{D}_{b}$ in $\mathcal{D}$ by $\mathcal{D}_{b}^{\prime}$. The proof that $\left(\mathcal{D}^{\prime}\right)^{t} \prec \mathcal{D}^{t}$ is analogous to the case $L \wedge$ for the existential quantifier above.

Suppose the last rule of $\mathcal{D}_{e}$ is $L \forall$. Then $S_{b}=\Pi, \forall y D y, \forall x B x \Rightarrow F$ and $\mathcal{D}_{b}$ ends as follows:

$$
\frac{\Pi, \forall y D y, \forall x B x, D t \Rightarrow F}{\Pi, \forall y D y, \forall x B x \Rightarrow F}
$$

If $s \neq t$, the case can be treated as before. Otherwise $\mathcal{D}_{b}$ ends as follows:

$$
\frac{\Pi, \forall y D y, \forall x B x, D t \Rightarrow F}{\Pi, \forall y D y, \forall x B x \Rightarrow F}
$$

In this case $\mathcal{D}^{\prime}$ is the result of replacing in $\mathcal{D}$ the derivation $\mathcal{D}_{e}$ by the subproof of the rightmost sequent $\Gamma, \forall y D y, \forall x B x \Rightarrow E t$. Clearly $\left(\mathcal{D}^{\prime}\right)^{t} \prec \mathcal{D}^{t}$. 
Theorem 2 If there is at most one occurrence of $A t \wedge E t$ in $S$, where $t \notin \mathcal{L}$, $A t \wedge E t$ occurs negatively, and $t$ occurs in $S$ only in this formula, then

$$
\vdash_{\mathcal{L}} S^{\exists x A x} \text { if and only if } \vdash_{\mathcal{L}} S \text {. }
$$

Proof The direction from left to right is trivial. For the other direction, let $\mathcal{D}$ be an ecut-free proof of $S$. By Lemma 2 it suffices to consider the case in which $t_{b}=0$ for all branches in $\mathcal{D}$. We show that all $S_{b}^{\exists x A x}$ are derivable and after that explain that this implies the derivability of $S^{\exists x A x}$.

For every $S_{b}$ there are three possibilities, see section 5.2. In case 3., At $\wedge E t$ is nowhere introduced along $b$, then either it does not occur in the leaf $S_{b}$ of $b$, or it occurs as or in a side formula. In this case $S_{b}^{\exists x A x}$ clearly is derivable.

In case $2 ., S_{b}^{\prime}=\Gamma, A t \wedge E t \Rightarrow B$, and there is no application of a quantifier rule with main variable $t$ below $S_{b}^{\prime}$. By Remark $1, S_{b}=\Gamma, A t, E t \Rightarrow B$. Since there is no application of a quantifier rule with main variable $t$ below $S_{b}^{\prime}$, all occurrences of $t$ in $\Gamma$ and $B$ occur in a formula $A t \wedge E t$ that is an ancestor of the same formula in the endsequent. For since we consider ecut-free proofs, and $t$ is not in $\mathcal{L}$, terms containing $t$ will not be cut away by an ecut. Since $S_{b}$ is derivable, so is $\Gamma^{\top}, A t, E t \Rightarrow B^{\top}$. The term $t$ is not free in $\Gamma^{\top}$ and $B^{\top}$, and therefore $L \exists$ can be applied to obtain $\Gamma^{\top}, \exists x A x \Rightarrow B^{\top}$. Hence $\left(\Gamma^{\exists x A x}, \exists x A x \Rightarrow B\right)^{\exists x A x}$ is derivable.

In case 1., $S_{b}$ is the conclusion of an application of a quantifier rule with main variable $t$. Thus $S_{b}=\Gamma \Rightarrow C$ and its derivation is:

$$
\frac{\Gamma \Rightarrow B \quad \Gamma \Rightarrow E t}{\Gamma \Rightarrow C}
$$

Since $t_{b}=0, \Gamma \Rightarrow E t$ is an axiom. Since $t \notin \mathcal{L}, E t \in \Gamma$ or $\perp \in \Gamma$. In the latter case $S_{b}^{\exists x A x}$ clearly is derivable too, since it is an instance of $L \perp$. In the former case, note that below $S_{b}$ neither is there an introduction of $A t \wedge E t$, nor is there a quantifier rule with main variable $t$, and $E t$ cannot be removed by an ecut because $t$ is not in $\mathcal{L}$. Therefore $E t$ has to occur in the endsequent in a formula other than $A t \wedge E t$, which by assumption cannot be. This completes the three cases, showing that all $S_{b}^{\exists x A x}$ are derivable.

It remains to be shown that this implies the derivability of $S^{\exists x A x}$. We construct a proof of this sequent in the following way. For every $S_{b}$ we replace its subproof in $\mathcal{D}$ by a proof of $S^{\exists x A x}$. In the remaining part of $\mathcal{D}$ every sequent $S^{\prime}$ is replaced by $\left(S^{\prime}\right)^{\exists x A x}$, while the rules are left unchanged. The result is a valid proof of $S^{\exists x A x}$. For consider an application of a rule below all $S_{b}$ 's. It is of the form

$$
\frac{S_{2} \quad S_{3}}{S_{1}} R
$$

or a single hypothesis rule. Since there is no introduction of $A t \wedge E t$ below any $S_{b}$, this implies that

$$
\frac{S_{2}^{\exists x A x} S_{3}^{\exists x A x}}{S_{1}^{\exists x A x}} R
$$

is a valid inference as well. This proves the theorem. 
Corollary $4 \vdash_{\mathcal{L}} S$ if and only if $\vdash_{\mathcal{L}} S^{e}$.

Proof By repeated application of the previous theorem. Suppose that the formulas $\exists x_{1} A x_{1}, \ldots, \exists x_{n} A_{n} x_{n}$ are the negative occurrences of existential quantifiers in $S$ and $\exists x_{i} A_{i}$ is not a subformula of $\exists x_{j} A_{j}$ for $j<i$, and let $t_{1}, \ldots, t_{n}$ be the corresponding skolem terms in $S^{e}$. Then

$$
S=\left(\ldots\left(\left(S^{e}\right)^{\exists x_{1} A x_{1}}\right) \ldots\right)^{\exists x_{n} A_{n} x_{n}},
$$

and for any $0<i \leq n$, there is at most one occurrence of $A_{i} t_{i} \wedge E t_{i}$ in $\left(\ldots\left(\left(S^{e}\right)^{\exists x_{1} A x_{1}}\right) \ldots\right)^{\exists x_{i-1} A_{n} x_{i-1}}$, it occurs negatively, $t_{i} \notin \mathcal{L}$, and there is no occurrence of $t_{i}$ in this sequent outside $A_{i} t_{i} \wedge E t_{i}$. Therefore the previous theorem can be applied to obtain a proof of $\left(\ldots\left(\left(S^{e}\right)^{\exists x_{1} A x_{1}}\right) \ldots\right)^{\exists x_{i} A_{n} x_{i}}$.

\section{References}

[1] M. Baaz and R. Iemhoff Gentzen calculi for the existence predicate, Studia Logica 82(1), 2006. (p.7-23)

[2] M. Baaz and R. Iemhoff On the Skolemization of existential quantifiers in intuitionistic logic, Annals of Pure and Applied Logic 142(1-3), 2006 (p.269295)

[3] M. Baaz and R. Iemhoff On Skolemization in constructive theories, Journal of Symbolic Logic 73(3), 2008 (p.969-998)

[4] M. Baaz and A. Leitsch On Skolemization and proof complexity, Fundamenta Informaticae 20, 1994. (p.353-379)

[5] R. Dyckhoff and L. Pinto Proof search in constructive logics, Sets and proofs, Lond. Math. Soc. Lect. vol. 258, Cambridge University Press, 1999. (p.53-65)

[6] M. Fitting Herbrand's theorem for a modal logic, Logic and foundations of mathematics. Selected contributed papers of the 10th international congress of logic, methodology and philosophy of science, Kluwer, 1999, (p.219-225)

[7] J. Herbrand Recherches sur la théorie de la demonstration, $\mathrm{PhD}$ thesis University of Paris, 1930.

[8] D. Hilbert and P. Bernays Grundlagen der Mathematik II, 2nd edition, Springer Berlin Heidelberg New York, 1970.

[9] G.E. Mints An analogue of Hebrand's theorem for the constructive predicate calculus, Sov. Math. Dokl. 3, 1962. (p.1712-1715)

[10] G.E. Mints Skolem's method of elimination of positive quantifiers in sequential calculi, Sov. Math.,Dokl. 7(4), 1966. (p.861-864)

[11] G.E. Mints The Skolem method in intuitionistic calculi, Proc. Steklov Inst. Math. 121, 1972. (p.73-109)

[12] G.E. Mints Resolution strategies for the intuitionistic predicate logic, Constraint Programming. Proceedings of the NATO Advanced Study Institute, Comput. Syst. Sci. 131, Springer, 1994. (p.289-311)

[13] G.E. Mints Axiomatization of a Skolem function in intuitionistic logic, Formalizing the dynamics of information, Faller, M. (ed.) et al. CSLI Lect. Notes 91, 2000. (p.105-114)

[14] D.S. Scott Identity and existence in intuitionistic logic, Applications of sheaves, Proc. Res. Symp. Durham 1977, Fourman (ed.) et al. Lect. Notes Math. 753, 1979. (p.660-696) 
[15] N. Shankar Proof search in the Intuitionistic Sequent Calculus, Automated deduction-CADE-11, Lect. Notes Comput. Science 607, 1992. (p.522-536)

[16] T. Skolem Logisch-kombinatorische Untersuchungen über die Erfüllbarkeit oder Beweisbarkeit mathematischer Sätze nebst einem Theorem über dichte Mengen, Skrifter utgitt av Videnskapsselskapet i Kristiania, I, Mat. Naturv. Kl. 4, 1920. (p.1993-2002)

[17] C. Smoryński Elementary intuitionistic theories, Journal of Symbolic Logic 38, 1973. (p.102-134)

[18] C. Smoryński On axiomatizing fragments, Journal of Symbolic Logic 42, 1977. (p.530-544)

[19] A.S. Troelstra and H. Schwichtenberg Basic Proof Theory, Cambridge Tracts in Theoretical Computer Science 43, Cambridge University Press, 1996.

[20] M. Unterhalt Kripke-Semantik mit partieller Existenz, PhD-thesis, University of Münster, 1986. 